\title{
REVIEWS
}

MICROBIOME

\section{The microbiota of the respiratory tract: gatekeeper to respiratory health}

\begin{abstract}
Wing Ho Man ${ }^{1,2 *}$, Wouter A.A. de Steenhuijsen Piters ${ }^{1,3 *}$ and Debby Bogaert ${ }^{1,3}$
Abstract | The respiratory tract is a complex organ system that is responsible for the exchange of oxygen and carbon dioxide. The human respiratory tract spans from the nostrils to the lung alveoli and is inhabited by niche-specific communities of bacteria. The microbiota of the respiratory tract probably acts as a gatekeeper that provides resistance to colonization by respiratory pathogens. The respiratory microbiota might also be involved in the maturation and maintenance of homeostasis of respiratory physiology and immunity. The ecological and environmental factors that direct the development of microbial communities in the respiratory tract and how these communities affect respiratory health are the focus of current research. Concurrently, the functions of the microbiome of the upper and lower respiratory tract in the physiology of the human host are being studied in detail. In this Review, we will discuss the epidemiological, biological and functional evidence that support the physiological role of the respiratory microbiota in the maintenance of human health.
\end{abstract}

\section{Microbiota}

The microorganisms (including bacteria, archaea and single-celled eukaryotes and viruses that inhabit a particular niche.

\section{Anterior nares}

Openings in the nose that connect the external environment and the nasal cavity

${ }^{1}$ Department of Pediatric Immunology and Infectious Diseases, Wilhelmina Children's Hospital, University Medical Center Utrecht, Lundlaan 6, 3584 EA Utrecht, The Netherlands. 'Spaarne Gasthuis Academy, Spaarnepoort 1, 2134 TM Hoofddorp, The Netherlands. ${ }^{3}$ The University of Edinburgh/ MRC Centre for Inflammation Research, The Queen's Medical Research Institute, 47 Little France Crescent. Edinburgh EH16 4TJ, UK.

* These authors contributed equally to this work.

Correspondence to D.B.

D.Bogaert@ed.ac.uk
Microbial communities have co-evolved with humans and our ancestors for millions of years and they inhabit all surfaces of the human body, including the respiratory tract mucosa. Specific sites in the respiratory tract contain specialized bacterial communities that are thought to have a major role in the maintenance of human health. In the past decade, next-generation sequencing has led to major advances in our understanding of the possible functions of the resident microbiota. So far, research has largely focused on the gut microbiota and gut microbiota-derived metabolites, and their influence on host metabolism and immunity. However, recent studies on microbial ecosystems at other body sites, including the respiratory tract, reveal an even broader role for the microbiota in human health ${ }^{1}$.

The respiratory tract is a complex organ system that is divided into the upper respiratory tract (URT) and the lower respiratory tract (LRT). The URT includes the anterior nares, nasal passages, paranasal sinuses, the nasopharynx and oropharynx, and the portion of the larynx above the vocal cords, whereas the LRT includes the portion of the larynx below the vocal cords, the trachea, smaller airways (that is, bronchi and bronchioli) and alveoli. The primary function of the respiratory tract in human physiology is the exchange of oxygen and carbon dioxide. For this purpose, the adult human airways have a surface area of approximately $70 \mathrm{~m}^{2}$, which is 40 times larger than the surface area of the skin'2. This entire surface is inhabited by niche-specific bacterial communities, with the highest bacterial densities observed in the URT (FIG. 1). Over the years, evidence for the roles that bacterial communities in the URT have in preventing respiratory pathogens from establishing an infection on the mucosal surface and spreading to the LRT has accumulated. For most respiratory bacterial pathogens, colonization of the URT is a necessary first step before causing an upper, lower or disseminated respiratory infection ${ }^{3}$. Inhibition of this first step of pathogenesis for respiratory infections by the resident microbiota, a process that is also called 'colonization resistance', might be of paramount importance to respiratory health. Furthermore, if a pathogen has colonized the mucosal surface, it might be beneficial to both the microbial community and the host that these pathogens are kept at bay, preventing their overgrowth, inflammation and subsequent local or systemic spread ${ }^{4}$. In addition to this symbiotic relationship, the respiratory microbiota probably has a role in the structural maturation of the respiratory tract ${ }^{5}$ and in shaping local immunity ${ }^{6,7}$.

Current research questions address how the healthy respiratory microbiota is established and what ecological and environmental factors govern its development. Concurrently, the broad range of functions of the respiratory microbiome is starting to become clear. In this Review, we focus on the role that the respiratory microbiota has in the development and maintenance of human respiratory health.

Anatomical development and the microbiota Anatomical development and physiology. The development of the structures of the human respiratory tract is 


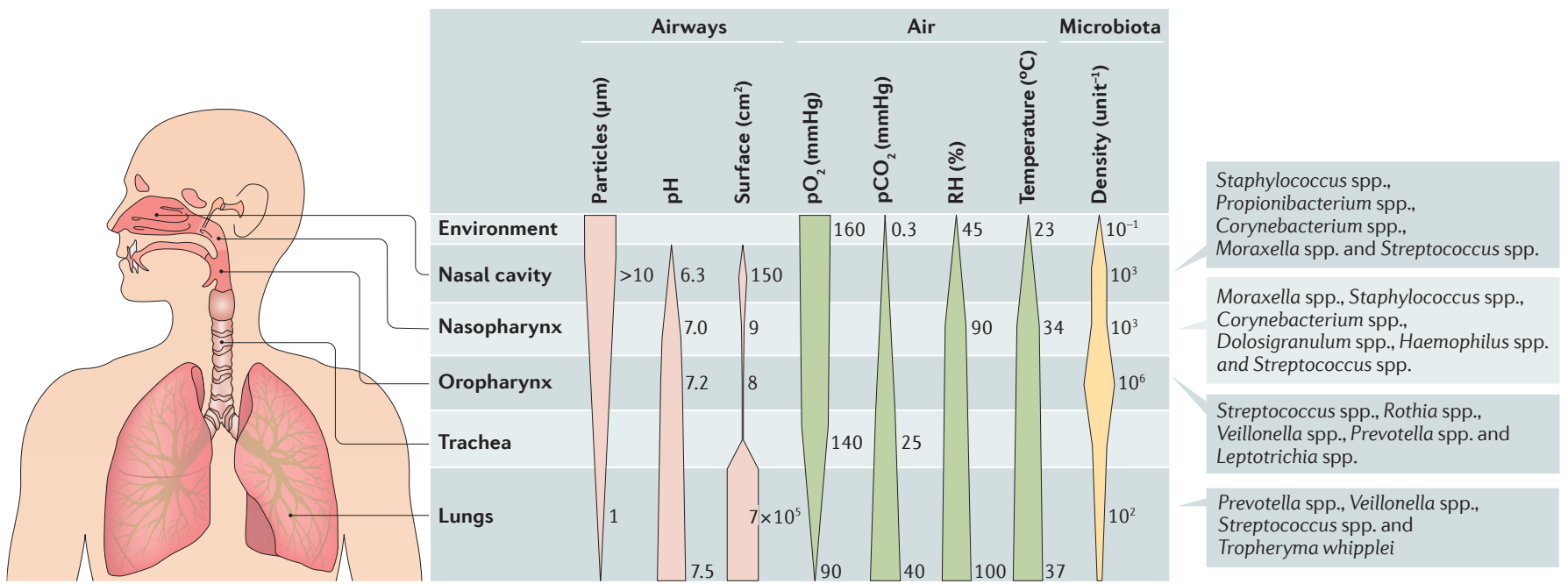

Figure 1 | Physiological and microbial gradients along the respiratory tract. Physiological and microbial gradients exist along the nasal cavity, nasopharynx, oropharynx, trachea and the lungs. The $\mathrm{pH}$ gradually increases along the respiratory tract ${ }^{177-180}$, whereas most of the increases in relative humidity $(\mathrm{RH})$ and temperature occur in the nasal cavity ${ }^{181-183}$. Furthermore, the partial pressures of oxygen $\left(\mathrm{pO}_{2}\right)$ and carbon dioxide $\left(\mathrm{pCO}_{2}\right)$ have opposing gradients ${ }^{180}$ that are determined by environmental air conditions and gas exchange at the surface of the lungs ${ }^{181,184,185}$. Inhalation results in the

Colonization

The act of settlement and

reproduction of organisms that are subject to selective pressure.

Symbiotic relationship A close biological interaction between two different species.

Microbiome

All of the genetic content of a microbial community.

Nasal placodes

A thickening of the embryonic head ectoderm that occurs in the fifth embryonic week and marks the start of the

formation of the nose and nasal cavity.

Oropharyngeal membrane A transient bilaminar (ectoderm and endoderm) membrane that appears in the fourth embryonic week during the development of the primitive mouth and pharynx.

\section{Lung buds}

A pair of endodermal outgrowths of the foregut that develop into the larynx, trachea and lungs.

Nasopharynx-associated lymphoid tissue

(NALT). One of the anatomical locations of mucosa-associated lymphoid tissue (MALT), which, in humans, consists of the lymphoid tissue of Waldeyer's pharyngeal ring, including the adenoids (the unpaired nasopharyngeal tonsil) and the paired palatine tonsils. deposition of particles from the environment into the respiratory tract; inhaled particles that are more than $10 \mu \mathrm{m}$ in diameter are deposited in the upper respiratory tract (URT), whereas particles less than $1 \mu \mathrm{m}$ in diameter can reach the lungs. These particles include bacteria-containing and virus-containing particles, which are typically larger than $0.4 \mu \mathrm{m}$ in diameter ${ }^{186}$. These physiological parameters determine the niche-specific selective growth conditions that ultimately shape the microbial communities along the respiratory tract. The unit by which bacterial density is measured varies per niche; the density in the environment is depicted as bacteria per $\mathrm{cm}^{3}$ (indoor) air ${ }^{187}$, density measures in the nasal cavity and nasopharynx are shown as an estimated number of bacteria per nasal $\mathrm{swab}^{74}$, and the densities in the oropharynx and the lungs represent the estimated number of bacteria per ml of oral wash ${ }^{57,74}$ or bronchoalveolar lavage $(B A L)^{57,74,153}$, respectively.

a complex multistage process that begins in the fourth week of gestation with the development of the nasal placodes, the oropharyngeal membrane and the lung buds 8.9 . The anatomy of the URT at birth is substantially different from the configuration in adults owing to the higher position of the larynx, which results in a large nasopharynx relative to the oropharyn $x^{10}$. In addition, the lack of alveoli in the newborn lungs underlines the immaturity of the LRT at birth. Indeed, the formation of alveoli begins in a late fetal stage and their development continues throughout the first three years of life ${ }^{11}$. By adulthood, many distinct subcompartments have developed in the respiratory tract, each of which has specific microbial, cellular and physiological features, such as oxygen and carbon dioxide tension, $\mathrm{pH}$, humidity and temperature (FIG. 1).

\section{Microbiota and the morphogenesis of the respiratory} tract. Similar to the anatomical development of the respiratory tract, the initial acquisition of microorganisms marks the establishment of the respiratory microbiota in early life. The establishment of the respiratory microbiota is thought to have an effect on the morphogenesis of the respiratory tract. Indeed, germ-free rodents tend to have smaller lungs ${ }^{12}$ and a decreased number of mature alveoli ${ }^{5}$. The latter finding was supported by experiments in which the nasal cavities of germ-free mouse pups were colonized with Lactobacillus spp., after which the number of mature alveoli normalized ${ }^{5}$. Intriguingly, the nasopharyngeal-associated lymphoid tissue (NALT) also develops mostly after birth, which suggests that its development requires environmental cues for example, from the local microbiota ${ }^{13}$.

Development of healthy microbiota. In contrast to the long-standing hypothesis that we are born sterile, it was recently suggested that babies acquire microorganisms in $u$ tero ${ }^{14,15}$, although this suggestion is controversial ${ }^{16}$. Irrespectively, the transfer of maternal antibodies and microbial molecules in utero markedly influences postnatal immune development ${ }^{17,18}$. This, in turn, primes the newborn for the substantial exposure to microorganisms that occurs after birth. During the first hours of life, a wide range of microorganisms can be detected in the URT of healthy term neonates ${ }^{19,20}$. At first, these microorganisms are nonspecific and are of presumed maternal origin. During the first week of life, niche differentiation in the URT leads to a high abundance of Staphylococcus spp., followed by the enrichment of Corynebacterium spp. and Dolosigranulum spp., and the subsequent predominance of Moraxella spp. ${ }^{20}$. Microbiota profiles that are characterized by Corynebacterium spp. and Dolosigranulum spp. early in life, and Moraxella spp. at 4-6 months of age, have been shown to correlate to a stable bacterial community composition and respiratory health ${ }^{21,22}$.

Birth mode and feeding type are important drivers of the early maturation of the microbiota, with children who are born vaginally and/or are breastfed transitioning 
towards a presumed health-promoting microbiota profile more often and more swiftly ${ }^{20,23}$. These findings were corroborated by epidemiological findings that showed breastfeeding-mediated protection against infections ${ }^{24}$, which is presumably a consequence of the transfer of maternal antibodies ${ }^{18}$ and beneficial microorganisms in breast milk, such as Bifidobacterium spp. and Lactobacillus spp. ${ }^{25,26}$. Conversely, the development of the respiratory microbiota can be disturbed, for example, through the use of antibiotics, which are commonly used in young children to treat infections ${ }^{27}$. Antibiotic perturbations were characterized by a decreased abundance of presumed beneficial commensal bacteria, such as Dolosigranulum spp. and Corynebacterium spp. in the URT of healthy children $22,28,29$. This, in turn, might increase the risk of respiratory tract infections following antibiotic treatment ${ }^{30}$. In addition, season, vaccination, presence of siblings, day-care attendance, exposure to smoke and prior infections can also affect the infant microbiota ${ }^{22,31-35}$, which indicates that the microbiota during early life is dynamic and affected by numerous host and environmental factors (FIG. 2). Host genetics seems to have a minor effect on the URT microbiota in healthy individuals, only influencing nasal bacterial density and not the composition of the microbiota ${ }^{36}$. By contrast, the composition of the sputum microbiota seems to be influenced equally by host genetics and environmental factors ${ }^{37}$.

Although the gut microbiota matures into an adultlike community during the first 3 years of life ${ }^{38}$, the time that is required to establish a stable respiratory microbiota remains to be determined. Although niche differentiation occurs as early as 1 week after birth ${ }^{20}$, the respiratory microbiota evolves throughout the first few years of life ${ }^{21,33,39}$. After the respiratory microbiota is established, antibiotic treatment remains an important perturbing factor of the microbial equilibrium throughout life ${ }^{40}$. Active smoking also affects the microbial communities in the URT ${ }^{37,41}$; however, in the LRT, smoking has no clear influence on the composition of the microbiota ${ }^{42}$. Interestingly, it has been suggested that the niche-specific differences disappear again in the elderly ${ }^{43}$.

Remarkably, not only exposure to beneficial bacteria seems to be important but also the timing of these exposures seems to play a crucial part in the maintenance of respiratory health, as especially aberrant respiratory colonization patterns in infancy seem to be a major determinant of respiratory disease later in life $\mathrm{f}^{21,22,44}$. This could be due to the effect of host-microbial interactions in immune education during early life ${ }^{6}$. It has been proposed that the dynamic nature of the developing microbiota early in life might provide a window of opportunity for the modulation of the microbiota towards a beneficial composition ${ }^{45}$; however, the extent of this period of time is currently unknown.

\section{The microbiota of the upper respiratory tract}

Gatekeeper to respiratory health. The URT consists of distinct anatomical structures that have different epithelial cell types and is exposed to various environmental factors. These diverse micro-niches are colonized by specialized bacterial communities, viruses and fungi.

The anterior nares are closest to the external environment and are lined with a skin-like keratinized squamous epithelium, including serous and sebaceous glands, the latter of which produces sebum, which leads to the enrichment of lipophilic skin colonizers, including Staphylococcus spp., Propionibacterium spp. and Corynebacterium spp. ${ }^{4-48}$. Bacteria that are frequently found in other respiratory niches, including Moraxella spp., Dolosigranulum spp. and Streptococcus spp., have also been observed in the anterior nares ${ }^{29,43,48,49}$. The nasopharynx is located deeper in the nasal cavity and

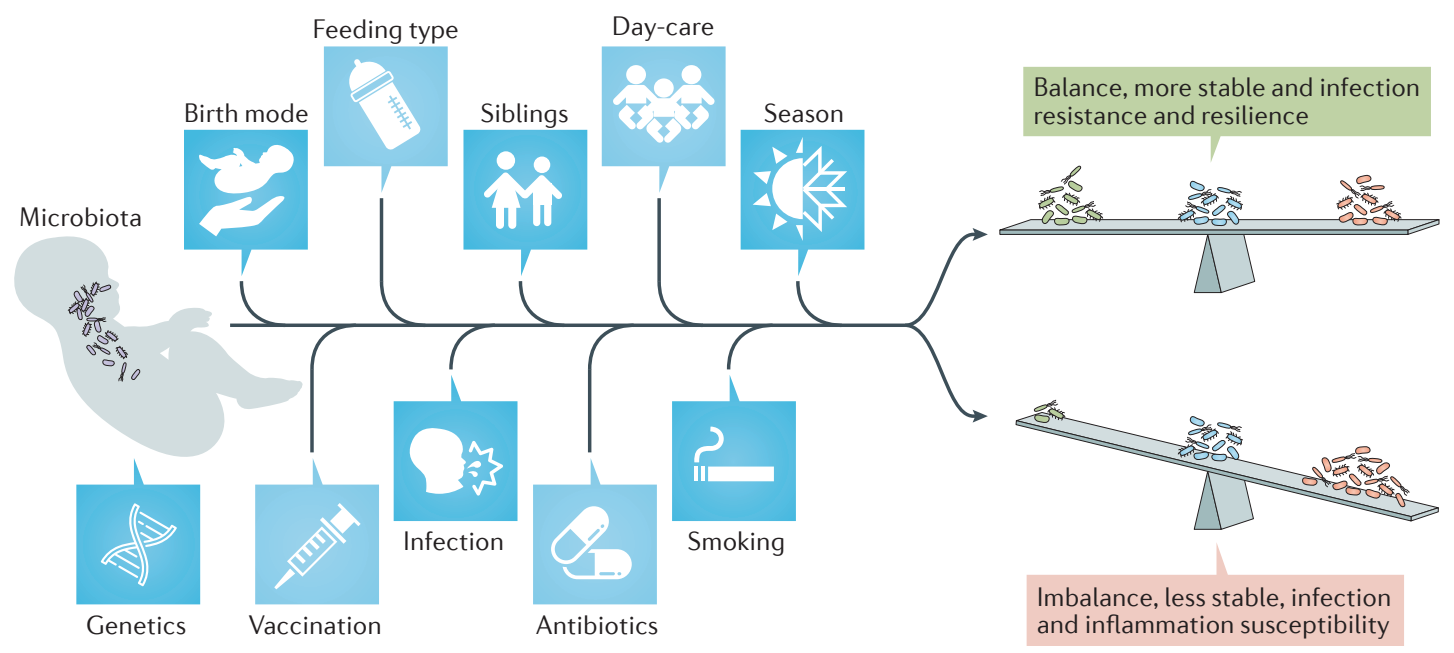

Figure 2 | Host and environmental factors that influence the respiratory microbiota. During early life, microbial communities in the respiratory tract are highly dynamic and are driven by multiple factors, including mode of birth, feeding type, crowding conditions and antibiotic treatment. Together, these host and environmental factors can change the composition of the microbiota towards a stable community at equilibrium that is resistant to pathogen overgrowth, or, conversely, an unstable community develops that is predisposed to infection and inflammation. 


\section{Biodiversity \\ The composite of species richness (the number of species present in an ecosystem) and evenness (the equitability of the abundance of these species).}

Acute otitis media (AOM). An acute-onset viral and/or bacterial infection of the middle ear.

Chronic rhinosinusitis

A common condition that is typified by prolonged

inflammation of the paranasal sinuses.

Keystone species A sole species that is typically not highly abundant but is disproportionally important in maintaining the organization and structure of an entire community.

Biofilms

Microorganisms embedded in a self-produced matrix of extracellular polymeric substances that are adherent to each other and/or a surface.

Bronchoalveolar lavages

(BALs). A technique in which fluid that contains bronchoalveolar cells is obtained by infusing and extracting saline during bronchoscopy.

Mucosal dispersion The separation and scattering of organisms from the mucosa.

Micro-aspiration Subclinical aspiration of small droplets. is covered by a stratified squamous epithelium that is punctuated by patches of respiratory epithelial cells. The composition of the bacterial communities in the nasopharynx is more diverse than in the anterior parts $\mathrm{s}^{50}$ and demonstrates considerable overlap with the anterior nares; it also contains Moraxella spp., Staphylococcus spp. and Corynebacterium spp. However, other bacteria more typically inhabit the nasopharyngeal niche, most notably Dolosigranulum spp., Haemophilus spp. and Streptococcus spp. ${ }^{20-22,33}$. The oropharynx, which is lined with a non-keratinized stratified squamous epithelium, has more diverse bacterial communities than the nasopharyn $\mathrm{x}^{41}$, which are characterized by streptococcal species, Neisseria spp., Rothia spp. and anaerobes, including Veillonella spp., Prevotella spp. and Leptotrichia spp. ${ }^{39,41,51,52}$.

In addition to bacterial inhabitants, PCR-based studies suggest the extensive presence of viral pathogens in the URT. These studies have reported an overall detection rate of $67 \%$ for respiratory viruses in healthy asymptomatic children, including human rhinovirus (HRV), human bocavirus, polyomaviruses, human adenovirus and human coronavirus ${ }^{31,53}$. However, recent advances in metagenomics have revealed that the entire respiratory virome contains many other viruses. For example, the recently discovered Anelloviridae family was identified as the most prevalent virus family in the virome of the URT ${ }^{54,55}$. Moreover, the healthy URT has a mycobiota that includes Aspergillus spp., Penicillium spp., Candida spp. and Alternaria spp. ${ }^{56,57}$. Although the size of the respiratory mycobiome is unknown, the gut and skin mycobiomes are approximated to comprise $0.1 \%$ and $3.9 \%$, respectively, of the total microbiome in their corresponding niches ${ }^{47,58}$.

Environmental pressures, as well as microorganismmicroorganism and host-microorganism interactions, influence the composition of the bacterial ecosystem in the human host and, as a consequence, its function. For various macroscale ecosystems, such as forests and coral reefs, it is well established that greater biodiversity increases the efficiency by which ecological communities are capable of using essential resources ${ }^{59}$. Similarly, the diversity of specific microscale ecosystems in the human host, such as the gut microbiota, has been associated with health outcomes. For example, increased diversity of intestinal bacteria has been linked to the absence of inflammatory bowel disease, obesity ${ }^{60}$ and resistance against acute infections by enteropathogen $s^{61}$. Conversely, at other body sites, such as the vagina, low bacterial diversity is considered 'healthy' as it is associated with decreased incidences of bacterial vaginosis ${ }^{62,63}$ and premature birth ${ }^{64}$, which highlights the niche-specific effect of biodiversity on human health. In the respiratory tract, evidence indicates that acute URT infections, such as acute otitis media $(\mathrm{AOM})^{29,65}$, and mucosal inflammation in chronic rhinosinusitis ${ }^{66}$ are associated with a decrease in the diversity of local bacterial communities. However, other studies report a less clear association between diversity and respiratory health, which suggests that the composition of bacterial communities, in a nichespecific ecological context, also affects respiratory health ${ }^{52}$. Moreover, certain members of the microbiota, known as 'keystone species', may have exceptionally large beneficial effects on ecosystem balance, function and health ${ }^{67}$. Potential keystone species in the URT microbiota are Dolosigranulum spp. and Corynebacterium spp., as they have been strongly associated with respiratory health and the exclusion of potential pathogens, most notably Streptococcus pneumoniae, in several epidemiological and mechanistic studies ${ }^{21,29,68,69}$.

A primary function of any microbial ecosystem is to elicit a state of symbiosis, providing 'colonization resistance' against pathogens $s^{4,70}$. The principal mechanism that underlies colonization resistance is that members of a diverse local microbiome probably use all of the nutrients that are available, thereby preventing pathogens from finding the necessary resources for colonization. Although cross-sectional surveys have demonstrated associations between decreased diversity and pathogen colonization, no direct evidence exists that demonstrates that increased microbial diversity in the respiratory tract can protect against the acquisition of pathogens. However, specific members of the microbiota have been identified that can actively exclude pathogens from the nasopharyngeal niche. For example, Staphylococcus epidermidis was shown to exclude Staphylococcus aureus and destroy pre-existing biofilms through the secretion of serine proteases $^{71}$. Furthermore, colonization resistance may be enhanced by interactions with the host immune system. For example, neutrophils seemed more able to kill S. pneumoniae following priming with Haemophilus influenzae ${ }^{72}$.

The URT is generally considered to be a major reservoir for potential pathogens, including S. pneumoniae, to expand and subsequently spread towards the lungs, which could potentially lead to a symptomatic infection ${ }^{3}$. Thus, establishing and maintaining a balanced microbiota in the URT that is resilient to pathogenic expansion and invasion could prove vital for respiratory health. The mechanisms that underlie a healthy respiratory microbiota, as well as specific microbiota-host interactions that support this, are considered below.

\section{Healthy lungs and their microbiota}

The LRT comprises the conducting airways (the trachea, bronchi and bronchioles) and the alveoli, in which gas exchange takes place. The conducting airways are lined with a similar respiratory epithelium to that found in the URT, with the epithelial cells gradually shifting towards a cuboidal shape along the respiratory tree. The alveoli in the lungs are lined with functionally distinct alveolar epithelial cells. In contrast to the URT and other human mucosal sites, the LRT has traditionally been considered as sterile; however, recent studies that used nextgeneration sequencing discovered a wide range of diverse microbial species in samples taken from the LRT. Potential contamination of low-density specimens remains a major concern when carrying out these types of study and caution is required when interpreting the results (BOX 1).

Source of the lung microbiota. In healthy individuals, bacteria enter the lungs by direct mucosal dispersion and micro-aspiration from the $\mathrm{URT}^{73}$. Culture-independent 


\section{Box 1 | Technical challenges in respiratory microbiome research}

Respiratory microbiome research faces general and niche-specific challenges. The absence of uniform laboratory practices (sample storage, DNA isolation and choice of $16 \mathrm{~S}$ rRNA variable region) and bioinformatics and data analysis pipelines limits the potential to carry out accurate comparative or meta-analyses. Although different research questions require different approaches, more energy should be invested into the development of standardized operating procedures that are comparable to, for example, the Earth Microbiome project protocol ${ }^{152}$.

A specific challenge in microbiota surveys of the respiratory tract is the low density of bacterial communities that are found there, particularly in healthy individuals; densities as low as $10^{2}-10^{3}$ bacteria $\mathrm{ml}^{-1}$ have been reported in bronchoalveolar lavages (BALs) of healthy individuals ${ }^{74,153}$. Such low quantities of DNA preclude whole-genome sequencing, which results in hindered taxonomic resolution and functional interpretation of microbiome data. Furthermore, sampling of the lower respiratory tract (LRT) is cumbersome and is typically based on BAL or the collection of expectorated sputum. Both sampling methods carry a high risk of cross-contamination of the LRT samples with resident bacterial communities in the upper respiratory tract (URT). Distinguishing between authentic LRT communities and URT contamination is further complicated because of the anatomical link between both niches. Once bacterial DNA has been extracted, the quantity can be so low that contamination from environmental DNA invalidates the results ${ }^{154,155}$. The development of standard operating procedures, including the careful use of appropriate negative controls at different stages of the sampling and laboratory workflow, can help to identify and exclude sequences from contaminating sources ${ }^{20,156}$.

Mutualism

An interaction between two species in which each species benefits (win-win).

Commensalism An interaction between two species in which one species benefits and the other is unaffected (win-neutral).

\section{Antagonism}

An interaction between two species in which one species is inhibited or adversely affected by another species, comprising: amensalism (lose-neutral), predatism and parasitism (win-lose) or competition (lose-lose).

Quorum sensing

A communication system between bacterial cells that is capable of triggering microbial group behaviour (for example, the formations of biofilms) once a certain threshold of signalling molecules is reached. microbiota studies have confirmed that the lung microbiota largely resembles the URT microbiota when studied in healthy individuals ${ }^{74-76}$. The oropharynx seems to be the main source of the lung microbiota in adults ${ }^{74}$, whereas in children the source is more likely to be both the nasopharynx and oropharyn $\mathrm{x}^{76}$. This might be due to the difference in the anatomy of the URT and the frequent increased production of nasal secretions in children, which both probably enhance the dispersal of microorganisms to the lungs. Another potential source of bacteria in the LRT is the direct inhalation of ambient air, albeit, to date, its direct influence on the lung microbiome is unknown. The contribution of the gastric microbiota to the microbial community in the LRT through gastric-oesophageal reflux has, until now, been suggested to be negligible ${ }^{74}$.

Composition of the lung microbiota. As LRT sampling is particularly challenging in young infants (BOX 1), current data on the composition and development of the neonatal LRT microbiota is limited to samples from intubated prematurely born infants $\mathrm{s}^{77-79}$. These studies showed that the LRT microbiota of premature infants is dominated by pathogenic Staphylococcus spp. ${ }^{78,79}$, Ureaplasma spp. ${ }^{79}$ or Acinetobacter spp. ${ }^{77}$, which highlights the lack of complexity in these developing bacterial communities.

In healthy children and adults, a unique microbial community in the lungs was found that contained many of the bacteria that are common to the URT. A study in young children reported that although the lung microbiota was distinct from the microbiota of the URT, it was dominated by species that are also present in the URT, including Moraxella spp., Haemophilus spp., Staphylococcus spp. and Streptococcus spp., but lacked other typical URT species, such as Corynebacterium spp. and Dolosigranulum spp. ${ }^{76}$. The adult lung microbiota seems to be dominated by genera in the phyla Firmicutes (including Streptococcus spp. and Veillonella spp.) and Bacteroidetes (including Prevotella spp. ${ }^{42,75,80}$. Interestingly, Tropheryma whipplei seems enriched only in the LRT, which suggests that this might be one of the few bacterial species that is not derived through dispersal from the URT ${ }^{42,75,80}$.

Studies of the LRT virome have revealed a high prevalence of members of the Anelloviridae family, in addition to a high frequency of bacteriophages ${ }^{81-83}$. Furthermore, the healthy lung mycobiome was found to be predominantly composed of members of the Eremothecium, Systenostrema and Malassezia genera, and the Davidiellaceae family, with common fungi in the URT detected only in low abundance ${ }^{57,84,85}$.

Although there are subtle regional variations of physiological parameters in the lungs (for example, of oxygen tension, $\mathrm{pH}$ and temperature), which, in theory, could affect microbial selection and growth, spatial microbial diversity in the lungs of healthy individuals seems almost absent ${ }^{75,80,86}$. This supports the hypothesis that, in health, the lung microbiota is a community of transiently present microorganisms that are derived from the URT, rather than a thriving, resident community as is commonly found in chronic respiratory diseases ${ }^{80,87,88}$. Correspondingly, a recently proposed ecological model, the adapted island model, postulates that the composition of a healthy lung microbiota is determined by the balance of microbial immigration and elimination ${ }^{80,88}$. Regardless, to date, the exact function that the lung microbiome has in establishing and maintaining respiratory health is unclear, although it probably contributes substantially to mucosal immune homeostasis (BOX 2).

\section{Interbacterial relationships}

Next-generation sequencing studies have revealed valuable information on both positive and negative microbial associations. By comparing sequencing data with mechanistic work, ecological interaction networks between microbial community members, or between the microbiota and the host or environment, can be partially reconstructed.

Associations between members of the microbiota can signify direct mutualism or commensalism (positive interactions), or antagonism (negative interactions). Positive interactions have been described primarily for members of the oropharyngeal microbiota; as such, Veillonella spp. were shown to induce streptococcal biofilm growth in a species-specific manner, presumably owing to shared quorum sensing systems ${ }^{89}$. These communication systems also seem to affect positive interactions between commensal and pathogenic members of the Streptococcus clade ${ }^{90}$, and between the nasopharyngeal community members Moraxella catarrhalis and $H$. influenzae ${ }^{91}$. Other mutualistic or commensal interactions in the nasopharyngeal microbiota exist, as illustrated by interactions between Corynebacterium spp. and Staphylococcus spp. The relationship between these species is complex and its directionality is probably species-specific or even strain-specific; Corynebacterium accolens and $S$. aureus mutually induce each other's 


\section{Box 2 | Specific host-microbiota interactions that contribute to tolerance}

The respiratory microbiota has been hypothesized to control mucosal immunity in early life and contribute to immune tolerance. For example, members of the Bacteroidetes phylum, such as Prevotella spp., decrease lung inflammation, neutrophil recruitment and the production of Toll-like receptor 2 (TLR2)-mediated pro-inflammatory cytokines compared with Haemophilus influenzae in a mouse model ${ }^{157}$, which could be related to the number of acyl side chains on their respective lipopolysaccharide (LPS) molecules ${ }^{158-160}$. Furthermore, in vitro activation of epithelial TLRs and nucleotide-binding oligomerization domain (NOD)-like receptors induced the release of antimicrobial peptides, such as $\beta$-defensin 2 (REF. 138), which could potentially influence the composition of the upper respiratory tract (URT) microbiota ${ }^{161}$. The production of these antimicrobial peptides is stimulated by Thelper $17\left(T_{H} 17\right)$ cells $^{162}$, which, in turn, were shown to be induced by specific microbial species ${ }^{163}$.

Intriguingly, immune signalling in the URT was shown to elicit responses in distally located mucosal tissues; intranasal inoculation of Staphylococcus aureus led to the TLR2-induced recruitment of monocytes to the lungs, in which they differentiated into immunosuppressive alveolar macrophages and subsequently dampened influenza virus-induced inflammatory responses ${ }^{164}$. Intranasal administration of Lactobacillus plantarum led to TLR2 and NOD2 receptor-mediated protection against lethal pneumovirus infection in the lungs of mice ${ }^{165}$. In addition, gut microbiota-induced priming of innate immune cells at the intestinal mucosa was shown to affect respiratory health; for example, through NOD1 receptor-mediated activation of the neutrophils that are required for the clearance of Streptococcus pneumoniae in the URT ${ }^{72,166}$. Furthermore, in germ-free mice, inoculation with microorganisms was shown to be essential for the recruitment of dendritic cells to the lungs ${ }^{167}$, and the priming of CD8 ${ }^{+}$ T cells ${ }^{168}$. Crosstalk between dendritic cells and T cells induces the release of immunoglobulin $\mathrm{A}(\lg \mathrm{A})$ at the mucosal interface, which prevents pathogens from interacting with the epithelium and selects for a heterogeneous composition of the gut microbiota, facilitating the expansion of regulatory T cells $\left(T_{\text {reg }} \text { cells }\right)^{135}$ (FIG. 3).

The most convincing evidence that the lung microbiota reciprocally affects local immune responses came from a study in healthy adults. In this study, specific lung bacteria (including Prevotella spp. and Veillonella spp.) were associated with an increased number of lymphocytes in bronchoalveolar lavage (BAL) fluid, $\mathrm{T}_{H} 17$ cell-mediated lung inflammation and a diminished TLR4 response by alveolar macrophages ${ }^{126}$.

Correspondingly, a positive correlation between the relative abundance of members of the phylum Proteobacteria and both alveolar and systemic inflammation was described for patients with acute respiratory distress syndrome (ARDS) ${ }^{169}$.

Regulatory T cells

( $\mathrm{T}_{\mathrm{reg}}$ cells). A subpopulation of $T$ cells that modulate the host immune system and are pivotal in the maintenance of

tolerance.

Acute respiratory distress syndrome

(ARDS). A clinical phenotype that occurs in patients who are critically ill and is characterized by overt lung inflammation in response to various

pathologies, including trauma, sepsis and pneumonia. growth through an unknown molecular mechanism ${ }^{50}$, whereas there are mixed reports about the interactions between Corynebacterium pseudodiphtheriticum and $S$. aureus ${ }^{46,50,92}$. Furthermore, antagonistic relationships have been identified, such as those between $S$. aureus and $S$. pneumoniae ${ }^{32}$, that may, in part, be explained by the production of pneumococcal hydrogen peroxide, which results in lethal bacteriophage induction in S. aureus $^{93,94}$. Human experimental colonization with the commensal Neisseria lactamica reduces existing Neisseria meningitidis colonization and even protects against new meningococcal acquisition, although the exact mechanisms that underlie this antagonistic relationship are unknown ${ }^{95}$.

It could be postulated that, especially in early life, the human host may nourish and promote specific members of the microbiota, such as $S$. aureus, to benefit from the wide range of antimicrobial molecules that it produces; this could aid the human host in its defence against invading pathogens ${ }^{96}$. The fact that $S$. aureus is present in almost all infants but only sporadically causes disease at this age could, in turn, be related to specific microbial interactions; for example, co-occurrence with Corynebacterium striatum was shown to increase the commensal behaviour of
S. aureus and decrease its virulence in an in vivo infection model ${ }^{17}$. Furthermore, interactions between species in the Staphylococcus genus might help to prevent $S$. aureus from overgrowing as well; for example, its colonization is hindered by serine protease activity in S. epidermidis ${ }^{71}$ and by the production of lugdunin by Staphylococcus lugdunensis, which is a natural antibiotic that is also active against other potential pathogens ${ }^{98}$.

Members of the microbiota might also modulate each other's growth in an indirect manner; for example, through outer membrane vesicle (OMV)-mediated immune evasion ${ }^{99}$, or by using specific properties of the local environment, as shown for C. accolens, which converts host triacylglycerols into free fatty acids (FFAs) that, in turn, limit pneumococcal growth ${ }^{68}$. A second example of these mechanisms is the frequent co-occurrence of Corynebacterium spp. and Dolosigranulum spp. in the nasopharynx ${ }^{20,22,69}$, in which Dolosigranulum spp. might be responsible for the acidification of the local environment, which, in turn, may facilitate the expansion of Corynebacterium spp.; however, a direct interaction between these species cannot be ruled out. Given the low density and presumably transient microbiota in the LRT, it could be speculated that the diversity of this microbiota is shaped by interbacterial relationships to a lesser extent than bacterial communities in the URT, although little is known about the level of proximity and likelihood of interbacterial effects.

Bacterial associations that are detected in epidemiological surveys may also indicate the existence of joint host or environmental drivers and not the presence of direct or indirect microbial interactions per se. For example, the co-occurrence of C. accolens and Propionibacterium spp. on the lipid-rich mucosa of the anterior nares ${ }^{48}$ could be explained by solely their joint lipophilic nature. Furthermore, epidemiological data suggest positive associations between $M$. catarrhalis, H. influenzae and S. pneumoniae that could be mediated by biological interactions or be based on their shared association with crowding conditions (for example, the presence of young siblings and day-care attendance) and/or their frequent asymptomatic co-presence with respiratory viruses ${ }^{53,100}$.

\section{Effect of the virome and mycobiome}

Bidirectional viral-bacterial interactions. Perhaps the best-known historical example of viral-bacterial interactions in the respiratory tract comes from the Spanish flu pandemic in 1918-1919, when millions of individuals died from secondary bacterial pneumonia after an initial infection with influenza $\mathrm{A}$ virus ${ }^{101}$. In addition, in the absence of disease, epidemiological studies have suggested the presence of viral-bacterial interactions (reviewed in REF. 102). The biological mechanisms that underlie these bidirectional interactions have been extensively studied, although mostly for viruses and bacteria that are known to cause respiratory diseases ${ }^{102}$.

One of the main modes of action by which respiratory viruses are thought to predispose individuals to bacterial disease is through the disruption of the airway-epithelial barrier, which facilitates the adhesion 
Toll-like receptor

An evolutionary conserved transmembrane protein that has a crucial role in innate immune responses against invading pathogens.

T helper 17 cells

$\mathrm{T}$ helper cells that are

characterized as preferential

producers of interleukin-17

(IL-17), mediate host defence

mechanisms to various

infections, and are involved in

the pathogenesis of several

autoimmune disorders.

Lipopolysaccharide

(LPS). The main constituent of the cell wall of Gram-negative bacteria and a potent Toll-like receptor 4 (TLR4) ligand. of bacterial pathogens ${ }^{103-105}$. Furthermore, it was demonstrated that influenza virus infection enhances colonization (especially by pneumococci) by liberating host-derived nutrients ${ }^{106}$ and by decreasing mucociliary clearance ${ }^{107}$. In addition, respiratory viruses can modulate innate and adaptive immune responses in the host, thereby promoting bacterial colonization and infection; for example, by impairing monocyte activity ${ }^{108}$, the extended desensitization of alveolar macrophages for Toll-like receptor (TLR)-ligands ${ }^{109}$, suppressing phagocytic capacity of alveolar macrophages ${ }^{110}$, and by inhibiting the production of antimicrobial peptides that is induced by T helper 17 cells $^{111}$.

Vice versa, respiratory bacteria can also promote viral infection through numerous pathways ${ }^{112-116}$. For example, the upregulation of adhesion receptors, such as intercellular adhesion molecule 1 (ICAM1), was shown to increase the binding of HRV and respiratory syncytial virus (RSV) to epithelial cells and amplify pro-inflammatory responses ${ }^{114-116}$. These findings were substantiated by a recent clinical study that showed that nasopharyngeal colonization by $S$. pneumoniae and $H$. influenzae in infants is associated with an amplified systemic RSV-induced host immune response, plausibly resulting in more severe RSV infection ${ }^{117}$.

Conversely, the presence of specific bacterial species in the respiratory microbiota may impede viral infections. These interactions can be either direct ${ }^{118,119}$ or indirect through the host immune system. For example, infection by influenza virus was shown to be less efficient following immune priming by lipopolysaccharide (LPS)mediated TLR4 activation of innate immune cells ${ }^{120,121}$.

\section{Box 3 | An early window of opportunity}

There is increasing evidence that early environmental and microbiota-derived cues are of paramount importance for the development of lymphoid tissue in neonates and ultimately shape the host immune system in the long term. For example, nasopharyngeal-associated lymphoid tissue (NALT) organogenesis is only initiated in the first week of life and is stimulated by cholera toxin, which suggests that NALT organogenesis may require microbiota-derived signals ${ }^{13}$. Similarly, the exposure of neonatal mice to lipopolysaccharide (LPS) led to the formation of bronchus-associated lymphoid tissue (BALT), which was not observed when mice were only exposed later in life $\mathrm{e}^{170}$. Furthermore, it was demonstrated that neonatal, but not adult, bacterial colonization attracts activated regulatory $T$ cells ( $T_{\text {reg }}$ cells) to the skin and is necessary for the induction of immune tolerance to skin commensals ${ }^{171}$. Similarly, the lung microbiota promotes the transient expression of programmed death ligand 1 (PDL1) in dendritic cells during the first two weeks of life, which is necessary for the $\mathrm{T}_{\text {reg }}$ cell-mediated attenuation of allergic airway responses ${ }^{7}$. Furthermore, hypermethylation of the CXC-motif chemokine ligand 16 ( $C x c l 16)$ gene in the lungs of germ-free mice increases the expression of CXCL16 and the accumulation of invariant natural killer T cells (NKT cells), which are known for their role in inflammation and asthma ${ }^{6}$. Transplantation of the microbiotas from normal mice at neonatal, but not adult age, prevented the accumulation of NKT cells, which abrogated disease in these mice (FIG. 3).

Altogether, these data suggest that the presence of a respiratory microbiota within a specific developmental period is crucial for shaping the adaptive immune response to commensals in adult life and coordinating the delicate balance between host, microbiota and the environment towards a long-term equilibrium. Although this early period of development can be regarded as a susceptible window for aberrant microbial colonization that could lead to the induction of immune disorders, importantly, this same phase of development may also provide a window of opportunity to intervene.

In fact, some studies suggest that LPS signalling is necessary for appropriate immune crosstalk and immune 'readiness' for future encounters with viruses ${ }^{122,123}$.

In general, the infection of bacteria by bacteriophages seems to be omnipresent. This phenomenon has even resulted in the evolution of a diverse range of antiviral defence mechanisms in commensal bacteria ${ }^{124}$. Consequently, selective infection of specific bacterial strains may regulate the composition of the bacterial community and may facilitate the adaptation of the bacterial community to novel environments by preserving its diversity ${ }^{125}$. A recent study also reported a broad overlap between species-specific bacteriophages and the bacterial community diversity in the lungs, which suggests that substantial interactions between the microbiota and bacteriophages exist in the healthy respiratory tract as well ${ }^{126}$.

Fungal-bacterial interactions. Mechanistic insight into the interactions between fungi, bacteria and the host during health is scarce. However, it has been demonstrated in vitro and in vivo that the formation of biofilms by $S$. aureus, Streptococcus spp. and P. aeruginosa damages respiratory epithelia, which enables fungal biofilms to develop ${ }^{127-129}$. Furthermore, $P$. aeruginosa stimulates the growth of Aspergillus fumigatus through sensing volatile metabolites at a distance ${ }^{130}$. Conversely, Candida albicans was shown to increase the prevalence of $P$. aeruginosa in mice by impeding the production of reactive oxygen species (ROS) by alveolar macrophages ${ }^{131}$. To date, the exact role and breadth of the mechanisms by which fungi contribute to a healthy equilibrium in the respiratory tract have unfortunately remained unstudied.

Although studies suggest the importance of both the respiratory virome and mycobiome in respiratory health, there is a considerable knowledge gap in their exact contributions to health compared with the role of the bacterial microbiome. However, current evidence provides an important basis for further in-depth analyses of the interactions that exist between bacteria, viruses and fungi, as well as the effect of host and environmental factors on these interactions.

\section{Host-bacterial interactions}

As there are a vast number of commensals and potential pathogens that inhabit the mucosa of the respiratory tract, a delicate equilibrium has to be maintained between immune sensing and tolerance of nonpathogenic commensals, and the containment of resident pathogens and new invaders. This fine balance is of specific importance to the LRT, as gaseous exchange is absolutely essential for human life and the lungs are exceptionally susceptible to damage from inflammatory responses. Below, we will provide an overview of the immune components that have a role in immune homeostasis in the URT and lungs. A detailed discussion of host-bacterial interactions and their role in immune homeostasis, organogenesis and immune education is provided in BOXES 2,3, respectively. In addition to bacteria, viruses may also promote host immune homeostasis (discussed in BOX 4). 


\section{Box 4 | Viral-host interactions}

Persistent viral infections naturally occur in humans and may regulate innate and adaptive immunity. In serum, there is an estimated daily turnover of more than $10^{9}$ anellovirus particles, which is thought to induce continuous immune surveillance and influence inhabitation by other microorganisms ${ }^{172}$. Similarly, chronic infection with herpesviruses, which have co-evolved with mammals for millions of years ${ }^{173}$ and can be detected in more than $90 \%$ of humans ${ }^{172}$, protects against bacterial infections by increasing the basal expression of interferon- $\gamma$ (IFN $\gamma$ ) and facilitating the activation of macrophages ${ }^{174}$. Likewise, acute infection with common respiratory viruses activates innate immune pathways that remain active after the virus has been cleared. For example, infection with Sendai virus in mice is associated with the interleukin-13 (IL-13)-dependent activation of natural killer T cells (NKT cells) and lung macrophages and subsequent airway hyper-reactivity ${ }^{175}$. Similarly, early infection with respiratory syncytial virus (RSV) leads to impaired regulatory $T$ cell ( $T_{\text {reg }}$ cell) function in mice, which increases the risk of allergic airway disease ${ }^{169}$. These findings are further supported by data from a human infant cohort study, in which persisting immune dysregulation was still detected one month after acute RSV infection ${ }^{176}$.

Inhabitation

The presence or occupancy of organisms.

\section{Mucus}

A viscous secretion that is

produced by goblet cells and is composed of a diverse range of mucin proteins.

Mucin

A class of gel-forming

glycoproteins that give mucus its viscosity.

Alveolar surfactant

A mixture of proteins and lipids

that reduce surface tension

and prevent alveolar collapse,

and, additionally, have

antimicrobial and

anti-inflammatory properties.

Lamina propria

A layer of loose connective

tissue that is located directly underneath the epithelium.

$\beta$-Defensin 2

An antimicrobial peptide that is produced by epithelial cells in the respiratory tract

following microbial stimulation.

Nucleotide-binding oligomerization domain-like receptors

(NOD-like receptors).

Intracellular, innate pattern recognition receptors that

recognize molecular fragments in peptidoglycan (a constituent of the bacterial cell wall).
The respiratory tract is exposed to large quantities of airborne particles from the environment. The first line of defence is the mucus layer of the nasopharynx and conducting airways. The mucus traps these particles, including microbial pathogens, which are then cleared through ciliary action towards the oral cavity. In addition, the glycoproteins in the mucus accommodate resident microorganisms and prevent infection ${ }^{132}$, as evidenced by the decrease in antibacterial cytokines and the presence of phagocytosis-impaired macrophages in the lungs of mucin-deficient mice ${ }^{133}$.

The mucus layer contains immunoglobulin A (IgA) produced by activated $B$ cells ${ }^{134}$ and can preclude pathogens from inhabiting the mucosal surface and interacting with epithelial surface receptors. IgA is also hypothesized to be involved in the regulation and selection of commensal microorganisms and establishing mutualistic host-microbial interactions ${ }^{135,136}$. Similarly, alveolar surfactant has an important role in lung innate immunity, as a deficiency in surfactant protein A has been associated with decreased bacterial phagocytosis and killing by alveolar macrophages ${ }^{137}$.

The next line of defence is the epithelial cell layer, which is essential for the spatial segregation of the microbiota and the underlying lamina propria. The respiratory tract epithelium produces various antimicrobial substances that contribute to barrier function, including human $\beta$-defensin 2 (REF. 138). Pharyngeal and lung epithelial cells, as well as macrophages and dendritic cells, have various receptors to sense the microbiota, including innate pattern recognition receptors (PRRs), such as TLRs and nucleotide-binding oligomerization domain-like receptors (NOD-like receptors) ${ }^{138}$, which are central to balancing the activation of downstream inflammatory signalling and the maintenance of immune tolerance. The epithelium in the URT is supported by mucosa-associated lymphoid tissue (MALT), which is populated with microfold cells that transport microorganisms from the epithelium to the lamina propria, where they can activate dendritic cells ${ }^{139}$. In the lungs, dendritic cells are located within and directly below the alveolar epithelium, where they continuously sample the alveolar space ${ }^{140}$. They subsequently present processed antigens to different subsets of $\mathrm{T}$ cells in the lung-draining lymph node, which initiates adaptive immune responses.

Anti-inflammatory alveolar macrophages are vital in lung immune homeostasis and for regulating the crosstalk between epithelial cells, dendritic cells and T cells (reviewed in REFS 141,142). These cells dampen TLR-induced inflammatory signals in epithelial cells ${ }^{143}$, suppress inflammation by inhibiting dendritic cellmediated activation of T cells ${ }^{144,145}$ and induce regulatory cells $^{146}$ (FIG. 3).

In conclusion, host-microbiota interactions influence different aspects of immune system development and contribute to immune maturation, immune tolerance and resistance to bacterial infection.

\section{Conclusions and perspectives}

The development of massive parallel sequencing ${ }^{147}$ has provided us with extensive insights into the microbial ecology of human body habitats, including the respiratory tract. Studies have shown that different ecological niches in the respiratory tract are occupied by diverse microbial communities that could act as gatekeepers to respiratory health. Further studies will be required to understand the pressures that shape these communities, their precise functions and contributions to human health. Efforts should focus on reductionist approaches to understand the underlying mechanisms involved in environment-microorganism, microorganismmicroorganism and microorganism-host interactions in their authentic ecological context. The use of in vitro models that enable the manipulation of specific bacterial, host or environmental factors could substantially advance our understanding of the respiratory microbiota. In addition, in vivo optical imaging techniques will help to visualize host-microbiota or intra-microbiota interactions in their spatial context in health and disease ${ }^{148}$. Data derived from these approaches could be used in mathematical models to reconstruct bacterial interactions and study host and environmental forces that govern microbial behaviour ${ }^{149}$.

In addition to the in-depth studies of highly complex and context-dependent interspecies and host-microbiota interaction networks, holistic approaches remain important. Although studies on the composition of the respiratory tract microbiota did not show substantial differences between different developed countries, the question as to whether comparable host and environmental factors regulate the respiratory microbiota of individuals living in low/middle-income countries remains an important open question. The high burden of infectious and inflammation-related diseases in developing areas of the world might at least, in part, be related to compositional changes in the respiratory microbiota and vice versa ${ }^{150}$. Most progress can be expected from large cohort studies, in which the microbiota of healthy individuals and individuals who have an increased risk of infectious respiratory diseases is longitudinally characterized. In parallel, multi-omic (for example, transcriptomic and metabolomic) and clinical data should be integrated to study the crosstalk between host and microorganism, 


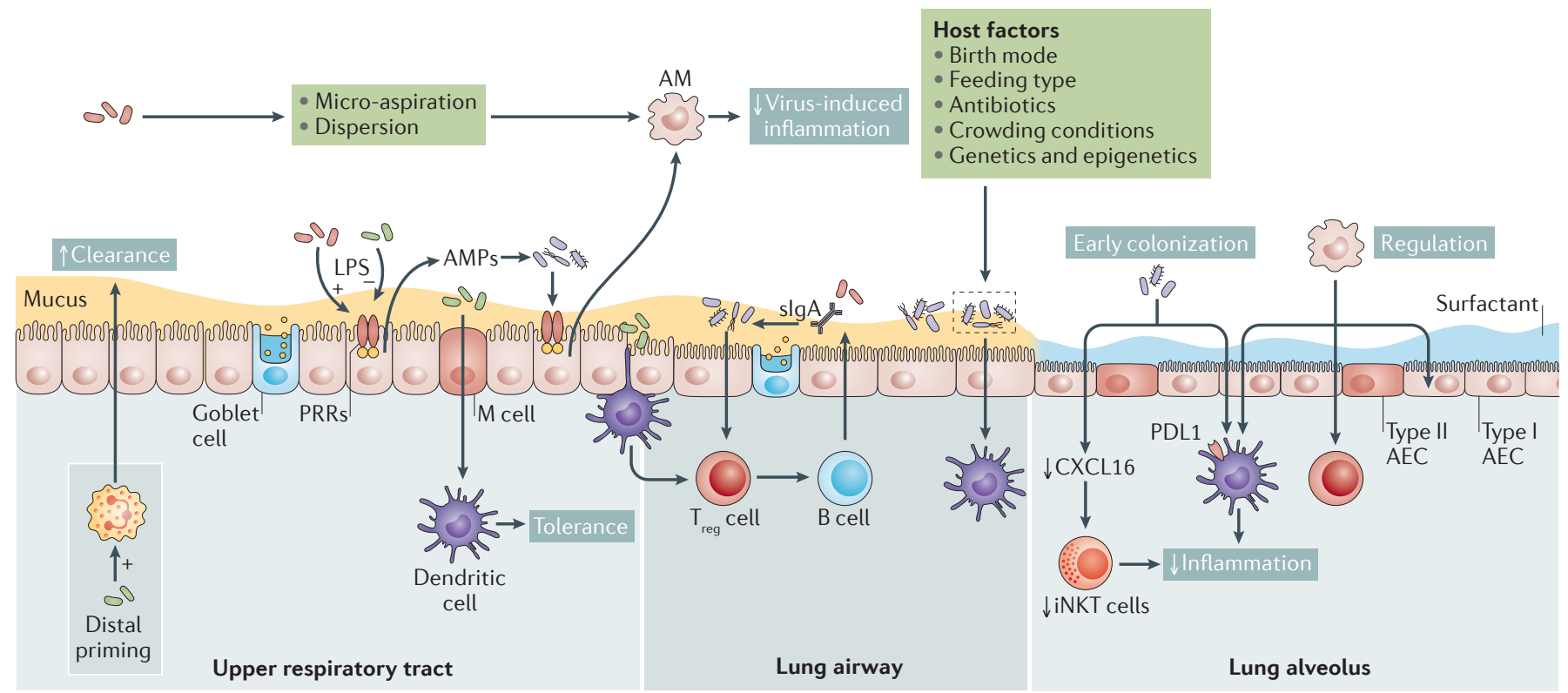

Figure 3 | Host-microbiota interactions in the respiratory tract. Host-microbiota interactions in the respiratory tract occur mostly at the mucosal surface. Resident microorganisms prime immune cells either locally or systemically; these include epithelial cells, neutrophils and dendritic cells, which all contribute to the clearance of pathogens. Moreover, microbial signalling is necessary for the recruitment and activation of regulatory cells, such as anti-inflammatory alveolar macrophages (AMs) and regulatory $T$ cells $\left(\mathrm{T}_{\text {reg }}\right.$ cells). Locally, the host will respond to microbial colonization through the release of antimicrobial peptides (AMPs) and secretory immunoglobulin A (slgA). Sensing of the microbiota involves microfold (M) cells that activate tolerogenic dendritic cells. In addition, alveolar dendritic cells can directly sample luminal microorganisms. Together, these pathways lead to the regulation of inflammation and the induction of tolerance, which, in turn, shape resident bacterial communities. It is also plausible that early bacterial colonization is key to long-term immune regulation, which is illustrated by the microbiota-induced decrease in hypermethylation of the CXC-motif chemokine ligand 16 (Cxcl16) gene, which prevents the accumulation of inducible natural killer T cells (iNKT cells), and by the programmed death ligand 1 (PDL1)-mediated induction of tolerogenic dendritic cells (BOX 3). This tolerant milieu, in turn, contributes to the normal development and maintenance of resident bacterial communities, which are also influenced by host and environmental factors (FIG. 1). AEC, alveolar epithelial cell; LPS: lipopolysaccharide; PRR: pattern recognition receptor; URT, upper respiratory tract.

microbiota function, and the effect of environmental factors on the composition of the microbiota. Consequently, advances in bioinformatics will be required to appropriately combine and analyse multiple high-dimensional datasets. Methods to analyse complex combinatorial data sets are sparse, but the field is rapidly progressing by applying machine-learning algorithms and time-resolved data modelling. A multidisciplinary approach to extract patterns and associations from these studies could culminate in individualized risk assessment and preventive personalized medicine, as illustrated by a study in which dietary interventions that were made based on the gut microbiota led to the improved control of post-meal glucose levels ${ }^{151}$. Microbiota-based interventions are likely to be most beneficial in young children, as a window of opportunity within which the local microbiota primes specific features of the immune system seems to exist. Interventions during this impressionable period could redirect an aberrant developmental route, potentially influencing long-term respiratory health.
1. Lloyd-Price, J., Abu-Ali, G. \& Huttenhower, C. The healthy human microbiome. Genome Med. 8, 51 (2016).

2. Weibel, E. R. Morphometry of the Human Lung (Springer Berlin Heidelberg, 1963).

3. Bogaert, D., De Groot, R. \& Hermans, P. W. M. Streptococcus pneumoniae colonisation: the key to pneumococcal disease. Lancet Infect. Dis. 4, 144-154 (2004).

This review discusses how nasopharyngeal colonization with S. pneumoniae can be an important prerequisite to respiratory and invasive pneumococcal disease.

4. Bäumler, A. J. \& Sperandio, V. Interactions between the microbiota and pathogenic bacteria in the gut. Nature 535, 85-93 (2016).

5. Yun, Y. et al. Environmentally determined differences in the murine lung microbiota and their relation to alveolar architecture. PLOS ONE 9, e 113466 (2014).
6. Olszak, T. et al. Microbial exposure during early life has persistent effects on natural killer $\mathrm{T}$ cell function. Science 336, 489-493 (2012). This study in mice highlights that the early presence of microbiota can lead to host epigenetic changes, decreased accumulation of pro-inflammatory cells and development towards an asthmatic phenotype later in life.

7. Gollwitzer, E. S. et al. Lung microbiota promotes tolerance to allergens in neonates via PD-L1. Nat. Med. 20, 642-647 (2014).

This paper comprehensively presents mechanistic evidence that the presence of the lung microbiota early in life promotes the healthy maturation of the neonatal immune system within a postnatal window of opportunity.

8. Som, P. M. \& Naidich, T. P. Illustrated review of the embryology and development of the facial region, part 1: early face and lateral nasal cavities. Am. J. Neuroradiol 34, 2233-2240 (2013).

9. Herriges, M. \& Morrisey, E. E. Lung development: orchestrating the generation and regeneration of a complex organ. Development 141, 502-513 (2014).

10. German, R. Z. \& Palmer, J. B. Anatomy and development of oral cavity and pharynx. GI Motil. Online http://www.nature.com/gimo/contents/pt1/full/ gimo5.html (2006).

11. Burri, P. H. Fetal and postnatal development of the lung. Annu. Rev. Physiol. 46, 617-628 (1984).

12. Wostmann, B. S. The germfree animal in nutritional studies. Annu. Rev. Nutr. 1, 257-279 (1981).

13. Fukuyama, S. et al. Initiation of NALT organogenesis is independent of the IL-7R, LT $\beta$ R, and NIK signaling pathways but requires the Id 2 gene and CD3-CD4 ${ }^{+} \mathrm{CD}_{4} 5^{+}$cells. Immunity 17, 31-40 (2002). 
14. Aagaard, K. et al. The placenta harbors a unique microbiome. Sci. Transl Med. 6, 237ra65 (2014)

15. Collado, M. C., Rautava, S., Aakko, J., Isolauri, E. \& Salminen, S. Human gut colonisation may be initiated in utero by distinct microbial communities in the placenta and amniotic fluid. Sci. Rep. 6, 23129 (2016).

16. Lauder, A. P. et al. Comparison of placenta samples with contamination controls does not provide evidence for a distinct placenta microbiota. Microbiome 4, 29 (2016).

17. Gomez de Agüero, M. et al. The maternal microbiota drives early postnatal innate immune development. Science 351, 1296-1302 (2016).

18. Koch, M. A. et al. Maternal IgG and IgA antibodies dampen mucosal $\mathrm{T}$ helper cell responses in early life. Cell 165, 827-841 (2016).

19. Dominguez-Bello, M. G. et al. Delivery mode shapes the acquisition and structure of the initial microbiota across multiple body habitats in newborns. Proc. Nat Acad. Sci. USA 107, 11971-11975 (2010).

20. Bosch, A. A. et al. Development of upper respiratory tract microbiota in infancy is affected by mode of delivery. EBioMedicine 9, 336-345 (2016).

21. Biesbroek, G. et al. Early respiratory microbiota composition determines bacterial succession patterns and respiratory health in children. Am. J. Respir. Crit. Care Med. 190, 1283-1292 (2014). This is a longitudinal study on the nasopharyngeal microbiota in children up to 2 years of age that links microbiome stability over time to environmental drivers, such as breastfeeding, specific bacterial community members and consecutive decreased rates of respiratory tract infections.

22. Teo, S. M. et al. The infant nasopharyngeal microbiome impacts severity of lower respiratory infection and risk of asthma development. Cell Host Microbe 17, 704-715 (2015)

This study in infants links nasopharyngeal colonization with Streptococcus spp., Moraxella spp. and Haemophilus spp. early in life to the development of LRT infections, consecutive atopic disease and future asthma.

23. Biesbroek, G. et al. The impact of breastfeeding on nasopharyngeal microbial communities in infants. $A m$ J. Respir Crit Care Med. 190, 298-308 (2014).

24. Duijts, L., Jaddoe, V. W. V., Hofman, A. \& Moll, H. A. Prolonged and exclusive breastfeeding reduces the risk of infectious diseases in infancy. Pediatrics 126 e18-e25 (2010).

25. Schanche, M. et al. High-resolution analyses of overlap in the microbiota between mothers and their children Curr. Microbiol. 71, 283-290 (2015)

26. Jeurink, P. V. et al. Human milk: a source of more life than we imagine. Benef. Microbes 4, 17-30 (2013).

27. Hicks, L. A., Taylor, T. H. \& Hunkler, R. J. U.S outpatient antibiotic prescribing, 2010. N. Engl. J. Med. 368, 1461-1462 (2013).

28. Prevaes, S. M. et al. Development of the nasopharyngeal microbiota in infants with cystic fibrosis. Am. J. Respir. Crit. Care Med. 193 504-515 (2016)

29. Pettigrew, M. M. et al. Upper respiratory tract microbial communities, acute otitis media pathogens, and antibiotic use in healthy and sick children. Appl. Environ. Microbiol. 78, 6262-6270 (2012).

This cohort study indicates that specific commensal nasopharyngeal bacteria, including Corynebacterium spp. and Dolosigranulum spp., are associated with the exclusion of pathogens that are known to cause acute otitis media (AOM) and the risk of AOM.

30. Leibovitz, E. et al. Recurrent acute otitis media occurring within one month from completion of antibiotic therapy: relationship to the original pathogen. Pediatr. Infect. Dis. J. 22, 209-216 (2003)

31. Bogaert, D. et al. Variability and diversity of nasopharyngeal microbiota in children: a metagenomic analysis. PLOS ONE 6, e1 7035 (2011).

32. Bogaert, D. et al. Colonisation by Streptococcus pneumoniae and Staphylococcus aureus in healthy children. Lancet 363, 1871-1872 (2004).

33. Mika, M. et al. Dynamics of the nasal microbiota in infancy: a prospective cohort study. J. Allergy Clin. Immunol. 135, 905-912.e11 (2015).

34. Spijkerman, J. et al. Long-term effects of pneumococcal conjugate vaccine on nasopharyngeal carriage of S. pneumoniae, S. aureus, H. influenzae and M. catarrhalis. PLoS ONE 7, e39730 (2012).

35. Greenberg, D. et al. The contribution of smoking and exposure to tobacco smoke to Streptococcus pneumoniae and Haemophilus influenzae carriage in children and their mothers. Clin. Infect. Dis. 42, 897-903 (2006)

36. Liu, C. M. et al. Staphylococcus aureus and the ecology of the nasal microbiome. Sci. Adv. 1, e1400216 (2015)

37. Lim, M. Y. et al. Analysis of the association between host genetics, smoking, and sputum microbiota in healthy humans. Sci. Rep. 6, 23745 (2016).

38. Yatsunenko, T. et al. Human gut microbiome viewed across age and geography. Nature 486, 222-227 (2012)

39. Stearns, J. C. et al. Culture and molecular-based profiles show shifts in bacterial communities of the upper respiratory tract that occur with age. ISME J. 9, 1246-1259 (2015).

40. Jakobsson, H. E. et al. Short-term antibiotic treatment has differing long-term impacts on the human throat and gut microbiome. PLOS ONE 5, e9836 (2010).

41. Charlson, E. S. et al. Disordered microbial communities in the upper respiratory tract of cigarette smokers. PLOS ONE 5, e15216 (2010).

42. Morris, A. et al. Comparison of the respiratory microbiome in healthy nonsmokers and smokers. Am. J. Respir. Crit. Care Med. 187, 1067-1075 (2013).

43. Whelan, F. J. et al. The loss of topography in the microbial communities of the upper respiratory tract in the elderly. Ann. Am. Thorac. Soc. 11, 513-521 (2014).

44. Vissing, N. H., Chawes, B. L. K. \& Bisgaard, H. Increased risk of pneumonia and bronchiolitis after bacterial colonization of the airways as neonates. $A m$ J. Respir. Crit. Care Med. 188, 1246-1252 (2013). This paper demonstrates that pharyngeal colonization by $S$. pneumoniae, $H$. influenzae or M. catarrhalis at $\mathbf{4}$ weeks of age is associated with an increased risk of respiratory tract infections during the first 3 years of life.

45. Dominguez-Bello, M. G. et al. Partial restoration of the microbiota of cesarean-born infants via vaginal microbial transfer. Nat. Med. 22, 250-253 (2016). In this proof-of-principle study, the investigators aim to restore the initial microbial communities of newborns that are delivered by caesarean section by transferring the maternal vaginal microbiota.

46. Frank, D. N. et al. The human nasal microbiota and Staphylococcus aureus carriage. PLOS ONE 5 e 10598 (2010)

47. Oh, J. et al. Biogeography and individuality shape function in the human skin metagenome. Nature $\mathbf{5 1 4}$, 59-64 (2014)

48. Zhou, Y. et al. Exploration of bacterial community classes in major human habitats. Genome Biol. 15 R66 (2014)

49. Wos-Oxley, M. L et al. Exploring the bacteria assemblages along the human nasal passage. Environ. Microbiol. 18, 2259-2271 (2016).

50. Yan, M. et al. Nasal microenvironments and interspecific interactions influence nasal microbiota complexity and $S$. aureus carriage. Cell Host Microbe 14, 631-640 (2013).

51. Segata, N. et al. Composition of the adult digestiv tract bacterial microbiome based on seven mouth surfaces, tonsils, throat and stool samples. Genome Biol. 13, R42 (2012).

52. de Steenhuijsen Piters, W. A. et al. Dysbiosis of upper respiratory tract microbiota in elderly pneumonia patients. ISME J. 10, 97-108 (2016).

53. van den Bergh, M. R. et al. Associations between pathogens in the upper respiratory tract of young children: interplay between viruses and bacteria. PLOS ONE 7, e47711 (2012)

This is the first study to investigate the prevalence and co-occurrence of potential bacterial respiratory pathogens and viruses in a large cohort of healthy children.

54. Wang, Y. et al. Metagenomic analysis of viral genetic diversity in respiratory samples from children with severe acute respiratory infection in China. Clin. Microbiol. Infect. 22, 458.e1-458.e9 (2016).

55. Wylie, K. M., Mihindukulasuriya, K. A., Sodergren, E., Weinstock, G. M. \& Storch, G. A. Sequence analysis of the human virome in febrile and afebrile children. PLoS ONE 7, e27735 (2012).

This study is the first to use metagenomics to assess the DNA and RNA virome in symptomatic and asymptomatic individuals, and reports that anelloviruses and enteroviruses are ubiquitous.

6. Eidi, S. et al. Nasal and indoors fungal contamination in healthy subjects. Health Scope 5 e30033 (2016)
57. Charlson, E. S. et al. Lung-enriched organisms and aberrant bacterial and fungal respiratory microbiota after lung transplant. Am. J. Respir. Crit. Care Med. 186, 536-545 (2012).

58. Qin, J. et al. A human gut microbial gene catalogue established by metagenomic sequencing. Nature 464 59-65 (2010)

59. Cardinale, B. J. et al. Biodiversity loss and its impact on humanity. Nature 486, 59-67 (2012).

60. Huttenhower, C., Kostic, A. D. \& Xavier, R. J. Inflammatory bowel disease as a model for translating the microbiome. Immunity 40, 843-854 (2014).

61. Kampmann, C., Dicksved, J., Engstrand, L. \& Rautelin, H. Composition of human faecal microbiota in resistance to Campylobacter infection. Clin. Microbiol. Infect. 22, 61.e1-61.e8 (2016).

62. Fredricks, D. N., Fiedler, T. L. \& Marrazzo, J. M. Molecular identification of bacteria associated with bacterial vaginosis. N. Engl. J. Med. 353, 1899-1911 (2005).

63. Ravel, J. et al. Vaginal microbiome of reproductive-age women. Proc. Natl Acad. Sci. USA 108, 4680-4687 (2011)

64. DiGiulio, D. B., Stevenson, D. K., Shaw, G., Lyell, D. J. \& Relman, D. A. Reply to Keelan and Payne: microbiota-related pathways for preterm birth. Proc. Natl Acad. Sci. USA 112, E6415 (2015).

65. Hilty, M. et al. Nasopharyngeal microbiota in infants with acute otitis media. J. Infect. Dis. 205, 1048-1055 (2012).

66. Abreu, N. A. et al. Sinus microbiome diversity depletion and Corynebacterium tuberculostearicum enrichment mediates rhinosinusitis. Sci. Transl Med. 4, 151 ra124 (2012).

67. Goodrich, J. K. et al. Human genetics shape the gut microbiome. Cell 159, 789-799 (2014)

68. Bomar, L., Brugger, S. D., Yost, B. H., Davies, S. S. \& Lemon, K. P. Corynebacterium accolens releases antipneumococcal free fatty acids from human nostril and skin surface triacylglycerols. mBio 7, e01725-15 (2016)

This in vitro study describes a mechanistic basis for the epidemiological association between Corynebacterium spp. and S. pneumoniae by demonstrating that $C$. accolens hydrolyses host triacylglycerols into free fatty acids that inhibit pneumococcal growth.

69. Laufer, A. S. et al. Microbial communities of the upper respiratory tract and otitis media in children. mBio 2 . e00245-10 (2011).

70. Kamada, N., Chen, G. Y., Inohara, N. \& Núñez, C. Control of pathogens and pathobionts by the gut microbiota. Nat. Immunol. 14, 685-690 (2013).

71. Iwase, T. et al. Staphylococcus epidermidis Esp inhibits Staphylococcus aureus biofilm formation and nasal colonization. Nature 465, 346-349 (2010).

72. Lysenko, E. S. et al. Nod 1 signaling overcomes resistance of $S$. pneumoniae to opsonophagocytic killing. PLoS Pathog. 3, e118 (2007).

73. Huxley, E. J., Viroslav, J., Gray, W. R. \& Pierce, A. K Pharyngeal aspiration in normal adults and patients with depressed consciousness. Am. J. Med. 64 564-568 (1978)

74. Bassis, C. M. et al. Analysis of the upper respiratory tract microbiotas as the source of the lung and gastric microbiotas in healthy individuals. $m B i o 6$, e00037-15 (2015)

75. Segal, L. N. et al. Enrichment of lung microbiome with supraglottic taxa is associated with increased pulmonary inflammation. Microbiome 1, 19 (2013).

76. Marsh, R. L. et al. The microbiota in bronchoalveolar lavage from young children with chronic lung disease includes taxa present in both the oropharynx and nasopharynx. Microbiome 4, 37 (2016)

77. Lohmann, P. et al. The airway microbiome of intubated premature infants: characteristics and changes that predict the development of bronchopulmonary dysplasia. Pediatr. Res. 76 294-301 (2014)

78. Payne, M. S. et al. Molecular microbiological characterization of preterm neonates at risk of bronchopulmonary dysplasia. Pediatr. Res. 67 412-418 (2010)

79. Mourani, P. M., Harris, J. K., Sontag, M. K Robertson, C. E. \& Abman, S. H. Molecular identification of bacteria in tracheal aspirate fluid from mechanically ventilated preterm infants. PLoS ONE 6, e25959 (2011).

80. Dickson, R. P. et al. Spatial variation in the healthy human lung microbiome and the adapted island model of lung biogeography. Ann. Am. Thorac. Soc. $12,821-830$ (2015) 
This paper demonstrates that spatial variation in the microbiota of healthy lungs is very minimal which suggests that the community composition in the LRT is determined principally by immigration and the elimination of microbial communities that originate from the URT.

81. Abbas, A. A. et al. The perioperative lung transplant virome: torque teno viruses are elevated in donor lungs and show divergent dynamics in primary graft dysfunction. Am. J. Transplant. http://dx.doi. org/10.1111/ajt. 14076 (2016)

82. Willner, D. et al. Metagenomic analysis of respiratory tract DNA viral communities in cystic fibrosis and noncystic fibrosis individuals. PLOS ONE 4, e7370 (2009).

83. Young, J. C. et al. Viral metagenomics reveal blooms of anelloviruses in the respiratory tract of lung transplant recipients. Am. J. Transplant. 15, 200-209 (2015).

84. van Woerden, H. C. et al. Differences in fungi present in induced sputum samples from asthma patients and non-atopic controls: a community based case control study. BMC Infect. Dis. 13, 69 (2013).

85. Cleland, E. J. et al. The fungal microbiome in chronic rhinosinusitis: richness, diversity, postoperative changes and patient outcomes. Int. Forum Allergy Rhinol. 4, 259-265 (2014).

86. Charlson, E. S. et al. Topographical continuity of bacterial populations in the healthy human respiratory tract. Am. J. Respir. Crit. Care Med. 184, 957-963 (2011).

87. Venkataraman, A. et al. Application of a neutral community model to assess structuring of the human lung microbiome. mBio 6, e02284-14 (2015).

88. Willner, D. et al. Spatial distribution of microbial communities in the cystic fibrosis lung. ISME J. 6 , 471-474 (2012)

89. Mashima, I. \& Nakazawa, F. The influence of oral Veillonella species on biofilms formed by Streptococcus species. Anaerobe 28, 54-61 (2014).

90. Cook, L. C., LaSarre, B. \& Federle, M. J. Interspecies communication among commensal and pathogenic streptococci. mBio 4, e00382-13 (2013).

91. Armbruster, C. E. et al. Indirect pathogenicity of Haemophilus influenzae and Moraxella catarrhalis in polymicrobial otitis media occurs via interspecies quorum signaling. mBio 1, e00102-10 (2010)

92. Wos-Oxley, M. L. et al. A poke into the diversity and associations within human anterior nare microbial communities. ISME J. 4, 839-851 (2010).

93. Regev-Yochay, G., Trzcinski, K., Thompson, C. M., Malley, R. \& Lipsitch, M. Interference between Streptococcus pneumoniae and Staphylococcus aureus: in vitro hydrogen peroxide-mediated killing by Streptococcus pneumoniae. J. Bacteriol. 188, 4996-5001 (2006).

94. Selva, L. et al. Killing niche competitors by remotecontrol bacteriophage induction. Proc. Natl Acad. Sci. USA 106, 1234-1238 (2009)

95. Deasy, A. M. et al. Nasal inoculation of the commensal Neisseria lactamica inhibits carriage of Neisseria meningitidis by young adults: a controlled human infection study. Clin. Infect. Dis. 60, 1512-1520 (2015).

96. Janek, D., Zipperer, A., Kulik, A., Krismer, B. \& Peschel, A. High frequency and diversity of antimicrobial activities produced by nasal Staphylococcus strains against bacterial competitors. PLoS Pathog. 12, e1005812 (2016).

97. Ramsey, M. M., Freire, M. O., Gabrilska, R. A., Rumbaugh, K. P. \& Lemon, K. P. Staphylococcus aureus shifts toward commensalism in response to Corynebacterium species. Front. Microbiol. 7, 1230 (2016).

98. Zipperer, A. et al. Human commensals producing a novel antibiotic impair pathogen colonization. Nature 535, 511-516 (2016).

99. Tong, T. T., Mörgelin, M., Forsgren, A. \& Riesbeck, K. Haemophilus influenzae survival during complementmediated attacks is promoted by Moraxella catarrhalis outer membrane vesicles. J. Infect. Dis. 195, 1661-1670 (2007).

100. de Steenhuijsen Piters, W. A., Sanders, E. A. $\delta$ Bogaert, D. The role of the local microbial ecosystem in respiratory health and disease. Philos. Trans. R. Soc B Biol. Sci. 370, 20140294 (2015).

101. Taubenberger, J. K., Reid, A. H. \& Fanning, T. G. The 1918 influenza virus: a killer comes into view. Virology 274, 241-245 (2000).

102. Bosch, A. A., Biesbroek, G., Trzcinski, K., Sanders, E. A. M. \& Bogaert, D. Viral and bacterial interactions in the upper respiratory tract. PLOS Pathog. 9, e1003057 (2013).
103. Sajjan, U., Wang, Q., Zhao, Y., Gruenert, D. C. \& Hershenson, M. B. Rhinovirus disrupts the barrier function of polarized airway epithelial cells. Am. J. Respir. Crit. Care Med. 178, 1271-1281 (2008).

104. Avadhanula, V. et al. Respiratory viruses augment the adhesion of bacterial pathogens to respiratory epithelium in a viral species- and cell type-dependent manner. J. Virol. 80, 1629-1636 (2006)

105. Ramphal, R., Small, P. M., Shands, J. W. Fischlschweiger, W. \& Small, P. A. Adherence of Pseudomonas aeruginosa to tracheal cells injured by influenza infection or by endotracheal intubation. Infect. Immun. 27, 614-619 (1980)

106. Siegel, S. J., Roche, A. M. \& Weiser, J. N. Influenza promotes pneumococcal growth during coinfection by providing host sialylated substrates as a nutrient source. Cell Host Microbe 16, 55-67 (2014).

107. Pittet, L. A., Hall-Stoodley, L., Rutkowski, M. R. \& Harmsen, A. G. Influenza virus infection decreases tracheal mucociliary velocity and clearance of Streptococcus pneumoniae. Am. J. Respir. Cell Mol. Biol. 42, 450-460 (2010).

108. Raza, M. W., Blackwell, C. C., Elton, R. A. \& Weir, D. M. Bactericidal activity of a monocytic cell line (THP-1) against common respiratory tract bacterial pathogens is depressed after infection with respiratory syncytial virus. J. Med. Microbiol. 49, 227-233 (2000).

109. Didierlaurent, A. et al. Sustained desensitization to bacterial Toll-like receptor ligands after resolution of respiratory influenza infection. J. Exp. Med. 205, 323-329 (2008)

110. Sun, K. \& Metzger, D. W. Inhibition of pulmonary antibacterial defense by interferon- $\gamma$ during recovery from influenza infection. Nat. Med. 14, 558-564 (2008)

111. Robinson, K. M. et al. Influenza A virus exacerbates staphylococcus aureus pneumonia in mice by attenuating antimicrobial peptide production. J. Infect. Dis. 209, 865-875 (2014).

112. Ni, K. et al. Pharyngeal microflora disruption by antibiotics promotes airway hyperresponsiveness after respiratory syncytial virus infection. PLOS ONE 7, e41104 (2012)

113. Wyde, P. R. Six H. R. Ambrose, M. W. \& Throop, B. J. Muramyl peptides and polyinosinic-polycytodylic acid given to mice prior to influenza virus challenge reduces pulmonary disease and mortality. J. Biol. Response Mod. 9, 98-102 (1990)

114. Sajjan, U. S. H. influenzae potentiates airway epithelial cell responses to rhinovirus by increasing ICAM-1 and TLR3 expression. FASEB J. 20 2121-2123 (2006)

115. Gulraiz, F., Bellinghausen, C., Bruggeman, C. A. \& Stassen, F. R. Haemophilus influenzae increases the susceptibility and inflammatory response of airway epithelial cells to viral infections. FASEB J. 29 849-858 (2015)

116. Bellinghausen, C. et al. Exposure to common respiratory bacteria alters the airway epithelial response to subsequent viral infection. Respir. Res. 17, 68 (2016)

117. de Steenhuijsen Piters, W. A. A. et al. Nasopharyngeal microbiota, host transcriptome and disease severity in children with respiratory syncytial virus infection. Am. J. Respir. Crit. Care Med. 1104-1115 (2016). This clinical study shows that the composition of the microbiota of the nasopharynx during early RSV infection is strongly associated with the differential expression of genes that are linked to innate immune pathways that, in turn, are associated with increased RSV disease severity.

118. Scheiblauer, H., Reinacher, M., Tashiro, M. \& Rott, R. Interactions between bacteria and influenza A virus in the development of influenza pneumonia. J. Infect. Dis. 166, 783-791 (1992)

119. Tashiro, M., Ciborowski, P., Klenk, H.-D., Pulverer, G. $\S$ Rott, R. Role of Staphylococcus protease in the development of influenza pneumonia. Nature 325 536-537 (1987).

120. Short, K. R. et al. Bacterial lipopolysaccharide inhibits influenza virus infection of human macrophages and the consequent induction of CD8 + T cell immunity. J. Innate Immun. 6, 129-139 (2014).

121. Ichinohe, T. et al. Microbiota regulates immune defense against respiratory tract influenza A virus infection. Proc. Natl Acad. Sci. USA 108, 5354-5359 (2011).

122. Amit, I. et al. Unbiased reconstruction of a mammalian transcriptional network mediating pathogen responses. Science 326, 257-263 (2009).
123. Abt, M. C. et al. Commensal bacteria calibrate the activation threshold of innate antiviral immunity. Immunity 37, 158-170 (2012)

This study highlights that LPS signalling of commensal bacteria contributes to immune 'readiness' and an adequate innate immune response following viral infection.

124. Pride, D. T., Salzman, J. \& Relman, D. A. Comparison of clustered regularly interspaced short palindromic repeats and viromes in human saliva reveal bacterial adaptations to salivary viruses. Environ. Microbiol. 14, 2564-2576 (2012)

125. Williams, H. T. P. Phage-induced diversification improves host evolvability. BMC Evol. Biol. 13, 17 (2013)

126. Segal, L. N. et al. Enrichment of the lung microbiome with oral taxa is associated with lung inflammation of a Th17 phenotype. Nat. Microbiol. 1, 16031 (2016).

127. Boase, S. et al. Bacterial-induced epithelial damage promotes fungal biofilm formation in a sheep model of sinusitis. Int. Forum Allergy Rhinol. 3, 341-348 (2013).

128. Diaz, P. I. et al. Synergistic interaction between Candida albicans and commensal oral streptococci in a novel in vitro mucosal model. Infect. Immun. $\mathbf{8 0}$ 620-632 (2012)

129. Xu, H. et al. Streptococcal co-infection augments Candida pathogenicity by amplifying the mucosal inflammatory response. Cell. Microbiol. 16, 214-231 (2014).

130. Briard, B., Heddergott, C. \& Latgé, J.-P. Volatile compounds emitted by Pseudomonas aeruginosa stimulate growth of the fungal pathogen Aspergillus fumigatus. mBio 7, e00219 (2016)

131. Roux, D. et al. Candida albicans impairs macrophage function and facilitates Pseudomonas aeruginosa pneumonia in rat. Crit. Care Med. 37, 1062-1067 (2009).

132. Linden, S. K., Sutton, P., Karlsson, N. G., Korolik, V. \& McGuckin, M. A. Mucins in the mucosal barrier to infection. Mucosal Immunol. 1, 183-197 (2008)

133. Roy, M. G. et al. Muc5b is required for airway defence. Nature 505, 412-416 (2014).

134. Kiyono, H. \& Fukuyama, S. NALT- versus Peyer's-patchmediated mucosal immunity. Nat. Rev. Immunol. 4 699-710 (2004)

135. Kawamoto, S. et al. Foxp3+ T cells regulate immunoglobulin A selection and facilitate diversification of bacterial species responsible for immune homeostasis. Immunity 41, 152-165 (2014).

136. Sutherland, D. B., Suzuki, K. \& Fagarasan, S. Fostering of advanced mutualism with gut microbiota by immunoglobulin A. Immunol. Rev. 270, 20-31 (2016).

137. LeVine, A. M. et al. Distinct effects of surfactant protein $\mathrm{A}$ or $\mathrm{D}$ deficiency during bacterial infection on the lung. J. Immunol. 165, 3934-3940 (2000).

138. Uehara, A., Fujimoto, Y., Fukase, K. \& Takada, H. Various human epithelial cells express functional Tolllike receptors, NOD1 and NOD2 to produce antimicrobial peptides, but not proinflammatory cytokines. Mol. Immunol. 44, 3100-3111 (2007).

139. Kim, D.-Y. et al. The airway antigen sampling system: respiratory $\mathrm{M}$ cells as an alternative gateway for inhaled antigens. J. Immunol. 186, 4253-4262 (2011).

140. Jahnsen, F. L. et al. Accelerated antigen sampling and transport by airway mucosal dendritic cells followin inhalation of a bacterial stimulus. J. Immunol. 177, 5861-5867 (2006)

141. Kopf, M., Schneider, C. \& Nobs, S. P. The development and function of lung-resident macrophages and dendritic cells. Nat. Immunol. 16, 36-44 (2014)

142. Hussell, T. \& Bell, T. J. Alveolar macrophages: plasticity in a tissue-specific context. Nat. Rev. Immunol. 14, 81-93 (2014).

143. Westphalen, K. et al. Sessile alveolar macrophages communicate with alveolar epithelium to modulate immunity. Nature 506, 503-506 (2014).

144. Holt, P. G. et al. Downregulation of the antigen presenting cell function(s) of pulmonary dendritic cells in vivo by resident alveolar macrophages. J. Exp. Med 177, 397-407 (1993).

145. Holt, P. G., Schon-Hegrad, M. A. \& Oliver, J. MHC class II antigen-bearing dendritic cells in pulmonary tissues of the rat. Regulation of antigen presentation activity by endogenous macrophage populations. J. Exp. Med. 167, 262-274 (1988).

146. Soroosh, P. et al. Lung-resident tissue macrophages generate Foxp3 ${ }^{+}$regulatory $T$ cells and promote airway tolerance. J. Exp. Med. 210, 775-788 (2013). 
147. Margulies, M. et al. Genome sequencing in microfabricated high-density picolitre reactors. Nature 437, 376-380 (2005)

148. Mills, B., Bradley, M. \& Dhaliwal, K. Optical imaging of bacterial infections. Clin. Transl Imaging 4, 163-174 (2016).

149. McLoughlin, K., Schluter, J., Rakoff-Nahoum, S., Smith, A. L. \& Foster, K. R. Host selection of microbiota via differential adhesion. Cell Host Microbe 19, 550-559 (2016)

150. Liu, L. et al. Global, regional, and national causes of child mortality: an updated systematic analysis for 2010 with time trends since 2000. Lancet $\mathbf{3 7 9}$, 2151-2161 (2012).

151. Zeevi, D. et al. Personalized nutrition by prediction of glycemic responses. Cell 163, 1079-1095 (2015).

152. Caporaso, J. G. et al. Ultra-high-throughput microbia community analysis on the Illumina HiSeq and MiSeq platforms. ISME J. 6, 1621-1624 (2012).

153. Goleva, E. et al. The effects of airway microbiome on corticosteroid responsiveness in asthma. Am. J. Respir. Crit. Care Med. 188, 1193-1201 (2013).

154. Salter, S. J. et al. Reagent and laboratory contamination can critically impact sequence-based microbiome analyses. BMC Biol. 12, 87 (2014).

155. Biesbroek, G. et al. Deep sequencing analyses of low density microbial communities: working at the boundary of accurate microbiota detection. PLOS ONE 7, e32942 (2012).

156. Dickson, R. P. et al. Enrichment of the lung microbiome with gut bacteria in sepsis and the acute respiratory distress syndrome. Nat. Microbiol. 1, 16113 (2016)

157. Larsen, J. M. et al. Chronic obstructive pulmonary disease and asthma-associated Proteobacteria, but not commensal Prevotella spp., promote Toll-like receptor 2-independent lung inflammation and pathology. Immunology 144, 333-342 (2015)

158. Bäckhed, F., Normark, S., Schweda, E. K. H. Oscarson, S. \& Richter-Dahlfors, A. Structural requirements for TLR4-mediated LPS signalling: a biological role for LPS modifications. Microbes Infect. 5, 1057-1063 (2003).

159. Coats, S. R., Reife, R. A., Bainbridge, B. W., Pham, T. T.-T. \& Darveau, R. P. Porphyromonas gingivalis lipopolysaccharide antagonizes Escherichia coli lipopolysaccharide at Toll-like receptor 4 in human endothelial cells. Infect. Immun. 71, 6799-6807 (2003).

160. Munford, R. S. Sensing Gram-negative bacterial lipopolysaccharides: a human disease determinant? Infect. Immun. 76, 454-465 (2008).

161. Salzman, N. H. et al. Enteric defensins are essentia regulators of intestinal microbial ecology. Nat. Immunol. 11, 76-83 (2010).
162. Kao, C.-Y. et al. IL-17 markedly up-regulates $\beta$-defensin-2 expression in human airway epithelium via JAK and NF- $\mathrm{KB}$ signaling pathways. J. Immunol. 173, 3482-3491 (2004)

163. Atarashi, K. et al. Th 17 cell induction by adhesion of microbes to intestinal epithelial cells. Cell 163 . 367-380 (2015)

164. Wang, J. et al. Bacterial colonization dampens influenza-mediated acute lung injury via induction of M2 alveolar macrophages. Nat. Commun. 4, 2106 (2013).

This study shows that nasal $S$. aureus instillation results in TLR2-dependent polarization towards anti-inflammatory alveolar macrophages, which attenuates influenza virus-induced immune-mediated lung injury.

165. Rice, T. A. et al. Signaling via pattern recognition receptors NOD2 and TLR2 contributes to immunomodulatory control of lethal pneumovirus infection. Antiviral Res. 132, 131-140 (2016).

166. Clarke, T. B. et al. Recognition of peptidoglycan from the microbiota by Nod 1 enhances systemic innate immunity. Nat. Med. 16, 228-231 (2010).

167. Herbst, T. et al. Dysregulation of allergic airway inflammation in the absence of microbia colonization. Am. J. Respir. Crit. Care Med. 184 198-205 (2011)

168. Naik, S. et al. Commensal-dendritic-cell interaction specifies a unique protective skin immune signature. Nature 520, 104-108 (2015).

169. Krishnamoorthy, N. et al. Early infection with respiratory syncytial virus impairs regulatory $\mathrm{T}$ cell function and increases susceptibility to allergic asthma. Nat. Med. 18, 1525-1530 (2012).

170. Rangel-Moreno, J. et al. The development of inducible bronchus-associated lymphoid tissue depends on IL-17. Nat. Immunol. 12, 639-646 (2011).

171. Scharschmidt, T. C. et al. A wave of regulatory T cells into neonatal skin mediates tolerance to commensal microbes. Immunity 43, 1011-1021 (2015).

172. Virgin, H. W., Wherry, E. J. \& Ahmed, R. Redefining chronic viral infection. Cell 138, 30-50 (2009).

173. McGeoch, D. J., Rixon, F. J. \& Davison, A. J. Topics in herpesvirus genomics and evolution. Virus Res. 117 90-104 (2006).

174. Barton, E. S. et al. Herpesvirus latency confers symbiotic protection from bacterial infection. Nature 447, 326-329 (2007)

175. Kim, E. Y. et al. Persistent activation of an innate immune response translates respiratory viral infection into chronic lung disease. Nat. Med. 14, 633-640 (2008).

176. Mejias, A. et al. Whole blood gene expression profiles to assess pathogenesis and disease severity in infants with respiratory syncytial virus infection. PLoS Med. 10, e1001549 (2013)

177. England, R. J., Homer, J. J., Knight, L. C. \& Ell, S. R. Nasal pH measurement: a reliable and repeatable parameter. Clin. Otolaryngol. Allied Sci. 24, 67-68 (1999).

178. Brunworth, J. D., Garg, R., Mahboubi, H., Johnson, B. \& Djalilian, H. R. Detecting nasopharyngeal reflux: a novel pH probe technique. Ann. Otol. Rhinol. Laryngol. 121, 427-430 (2012).

179. Ayazi, S. et al. A new technique for measurement of pharyngeal $\mathrm{pH}$ : normal values and discriminating $\mathrm{pH}$ threshold. J. Gastrointest. Surg. 13, 1422-1429 (2009).

180. West, J. B. Regional differences in the lung. Chest $\mathbf{7 4}$, 426 (1978).

181. Keck, T. \& Lindemann, J. Numerical simulation and nasal air-conditioning. GMS Curr. Top. Otorhinolaryngol. Head Neck Surg. http://dx.doi. org/10.3205/cto000072 (2010).

182. Ingenito, E. P. et al. Indirect assessment of mucosal surface temperatures in the airways: theory and tests. J. Appl. Physiol. 63, 2075-2083 (1987).

183. McFadden, E. R. et al. Thermal mapping of the airways in humans. J. Appl. Physiol. 58, 564-570 (1985).

184. Morgan, N. J., MacGregor, F. B., Birchall, M. A., Lund, V. J. \& Sittampalam, Y. Racial differences in nasal fossa dimensions determined by acoustic rhinometry. Rhinology 33, 224-228 (1995).

185. Walsh, J. H. et al. Evaluation of pharyngeal shape and size using anatomical optical coherence tomography in individuals with and without obstructive sleep apnoea. J. Sleep Res. 17, 230-238 (2008)

186. Hatch, T. F. Distribution and deposition of inhaled particles in respiratory tract. Bacteriol. Rev. 25 237-240 (1961)

187. Prussin, A. J. \& Marr, L. C. Sources of airborne microorganisms in the built environment. Microbiome 3, 78 (2015)

\section{Acknowledgements}

The authors apologize to all colleagues whose work could not be cited owing to space limitations. This work was supported by the Netherlands Organization for Scientific Research through NWO-Vidi (grant 91715359 to D.B.), a Scottish Senior Clinical Fellowship award (to D.B.), the Spaarne Gasthuis Academy Hoofddorp (to W.H.M.) and Wilhelmina Children's Hospital intramural funds (to W.A.A.d.S.P.). The authors thank colleagues in Utrecht, Hoofddorp and Edinburgh for in-depth discussions in regard to topics covered in this manuscript: these discussions have substantially contributed to the content of this work.

\section{Competing interests statement}

The authors declare no competing interests. 